\title{
Resveratrol attenuates steatosis in obese Zucker rats by decreasing fatty acid availability and reducing oxidative stress
}

\author{
S. Gómez-Zorita ${ }^{1,2}$, A. Fernández-Quintela ${ }^{1,2}$, M. T. Macarulla ${ }^{1,2}$, L. Aguirre ${ }^{1,2}$, E. Hijona ${ }^{3}$, L. Bujanda Ba $^{3,4}$, \\ F. Milagro 5 , J. A. Martínez ${ }^{2,5}$ and M. P. Portillo, ${ }^{1,2 *}$ \\ ${ }^{1}$ Department of Nutrition and Food Science, University of País Vasco, Vitoria, Spain \\ ${ }^{2}$ RETIC PREDIMED, Instituto de Salud Carlos III, Madrid, Spain \\ ${ }^{3}$ Department of Gastroenterology, Donostia Hospital, Centro de Investigación Biomédica en Enfermedades Hepáticas \\ (CIBERehd), San Sebastián, Spain \\ ${ }^{4}$ Department of Medicine, University of País Vasco, San Sebastián Madrid, Spain \\ ${ }^{5}$ Department of Nutrition and Food Sciences, Physiology and Toxicology, University of Navarra, Pamplona, Spain
}

(Received 16 November 2010 - Revised 1 April 2011 - Accepted 1 April 2011 - First published online 28 June 2011)

\section{Abstract}

Non-alcoholic fatty liver disease (NAFLD) is one of the most common manifestations of chronic liver disease worldwide. The aim of the present study was to assess the effect of resveratrol on liver fat accumulation, as well as on the activity of those enzymes involved in lipogenesis and fatty acid oxidation in $f a / f a$ Zucker rats. A total of thirty rats were assigned to three experimental groups and orally treated with resveratrol for 6 weeks, or without resveratrol (C: control group; RSV15 group: $15 \mathrm{mg} / \mathrm{kg}$ body weight per d; RSV45 group: $45 \mathrm{mg} / \mathrm{kg}$ body weight per d). Liver histological analysis was performed by microscopy. Levels of hepatic carnitine palmitoyltransferaseIa (CPT-Ia), acyl-coenzyme A oxidase (ACO), fatty acid synthase, glucose-6-phosphate dehydrogenase and malic enzyme were assessed by spectrophotometry, and acetyl-CoA carboxylase was assessed by radiometry. Commercial kits were used to determine serum TAG, NEFA, total HDL and non-HDL-cholesterol, glycerol, ketonic bodies, glucose, insulin, adiponectin, aspartate aminotransferase (AST), alanine aminotransferase (ALT) and alkaline phosphatase (ALP), hepatic TAG, thiobarbituric acid reactive substrates, GSH (GSSG) and superoxide dismutase. Resveratrol reduced liver weight and TAG content. It did not modify the activity of lipogenic enzymes but it did increase CPT-Ia and ACO activities. NEFA and ALP were reduced in both resveratrol-treated groups. AST/GOT was reduced only by the lowest dose. ALT/GPT, TAG and adiponectin remained unchanged. Resveratrol reduced liver oxidative stress. This study demonstrates that resveratrol can protect the liver from NAFLD by reducing fatty acid availability. Moreover, resveratrol also protects liver from oxidative stress.

\section{Key words: Resveratrol: Liver: Steatosis: Zucker rats: Lipogenesis: Fatty acid oxidation: Oxidative stress}

Non-alcoholic fatty liver disease (NAFLD) is one of the most common manifestations of chronic liver disorders worldwide $^{(1)}$. NAFLD represents a spectrum of hepatic dysfunctions that occur in the absence of alcohol consumption in amounts generally considered to be harmful to the liver. It is characterised by histological changes consisting in an increased accumulation of lipids into small droplets inside the cytoplasm, in hepatocytes. This phenomenon occurs when the rate of hepatic fatty acid uptake from plasma and de novo fatty acid synthesis are greater than that of fatty acid oxidation and release of TAG within VLDL ${ }^{(2)}$. NAFLD is closely associated with obesity and insulin resistance ${ }^{(3)}$.
There are many models of NAFLD in animals that have been used in many studies. One of these models is the obese Zucker rat. This animal strain shows an earlier obesity, which is accompanied by many of the human metabolic syndrome features and liver steatosis. Peripheral insulin resistance in obese Zucker rats enhances the mobilisation of peripheral fat and the serum level of NEFA. However, the liver oxidation or utilisation of NEFA is inhibited. Thus, the liver in this rodent model synthesises excess TAG and oxidises a small amount of fatty acids, leading to fat infiltration of the hepatic parenchyma ${ }^{(4)}$.

Abbreviations: ACC, acetyl-COA carboxylase; ALT, alanine aminotransferase; AST, aspartate aminotransferase; CPT-Ia, carnitine palmitoyltransferase-Ia; FAS, fatty acid synthase; G6PDH, glucose-6-phosphate dehydrogenase; NAFLD, non-alcoholic fatty liver disease; TBARS, thiobarbituric acid reactive substrates. 
Resveratrol (trans-3,5,4'-trihydroxystilbene) is a phytoalexin polyphenolic compound occurring naturally in various plants, including grapes, berries and peanuts, in response to stress and as a defence mechanism against fungal, viral, bacterial infections and damage from exposure to UV radiation ${ }^{(5,6)}$.

A remarkable range of biological functions have been ascribed to this molecule. For example, it acts as a cancer chemoprevention agent, as a powerful anti-inflammatory factor and as an antioxidant agent ${ }^{(7,8)}$. Cardiovascular properties of resveratrol, including inhibition of platelet aggregation and promotion of vasodilation by enhancing the production of $\mathrm{NO}$, have also been described ${ }^{(9)}$. More recently, it has been demonstrated that resveratrol can modify TAG metabolism in adipose tissue and liver ${ }^{(10-18)}$.

The aim of the present study was to assess the effect of two graded doses of resveratrol on liver fat accumulation in the $f a / f a$ Zucker rat model of steatosis. To determine some of the mechanisms underlying this effect, we also analysed the influence of this polyphenol on the activity of enzymes involved in lipogenesis and fatty acid oxidation.

\section{Materials and methods}

\section{Animals, diets and experimental design}

The experiment was conducted on thirty male Zucker ( $f a / f a$ ) rats aged 6 weeks (213 (SEM 4) g) purchased from Harlan Ibérica (Barcelona, Spain) and took place in accordance with the institution's guide for the care and use of laboratory animals. The rats were individually housed in polycarbonate metabolic cages (Tecniplast Gazzada, Buguggiate, Italy) and placed in an air-conditioned room $\left(22 \pm 2^{\circ} \mathrm{C}\right)$ with a $12 \mathrm{~h}$ light-dark cycle. After a $6 \mathrm{~d}$ adaptation period, rats were randomly distributed in three experimental groups of ten animals each, and fed on a standard laboratory diet (Panlab, Barcelona, Spain). Rats in the resveratrol groups were orally administered resveratrol $(15 \mathrm{mg} / \mathrm{kg}$ body weight per $\mathrm{d}$ in RSV15 group and $45 \mathrm{mg} / \mathrm{kg}$ body weight per d in RSV 45 group) through an orogastric catheter for 6 weeks. Resveratrol was diluted in $1 \mathrm{ml}$ ethanolic solution (20\%). Rats from control group ( $\mathrm{C}$ group) received only the vehicle. All animals had free access to food and water. Food intake and body weight were measured daily.

At the end of the experimental period and after a fasting period of $6-8 \mathrm{~h}$, animals were killed by cardiac exsanguination under anaesthesia by using an intraperitoneal injection of an overdose $(45 \mathrm{mg} / \mathrm{kg})$ of sodium pentobarbital. White adipose tissue from different anatomical locations (perirenal, epididymal, mesenteric and subcutaneous regions) and liver were dissected, weighed and immediately frozen.

\section{Steatosis assessment}

Total lipids were extracted from liver following the method described by Folch et al. ${ }^{(19)}$. The lipid extract was dissolved in isopropanol. TAG and cholesterol contents were measured by using commercial kits (Spinreact, Barcelona, Spain). Moreover, a histological study was performed on the livers.
Just after killing, a piece of liver was placed in $10 \%$ buffered formalin and subsequently embedded in paraffin. Liver sections were stained with haematoxylin and eosin using standard techniques. Sections were viewed without knowing the treatment group to which each animal belonged. Biopsies were classified into four grades depending on fat accumulation using Brunt et al. ${ }^{(20)}$ classification, assigning grade 0 when no fat was found in the liver, grade 1 when fat vacuoles were seen in less than $33 \%$ of hepatocytes, grade 2 when $33-66 \%$ of hepatocytes were affected by fat vacuoles and grade 3 when fat vacuoles were found in more than $66 \%$ of hepatocytes. Two experienced pathologists who were masked to the experiment evaluated all samples, and reached an agreement.

\section{Enzyme activities}

For lipogenic enzyme analysis, samples of liver $(500 \mathrm{mg})$ were homogenised in $5 \mathrm{ml}$ buffer ( $\mathrm{pH} \mathrm{7.6)} \mathrm{containing} 150 \mathrm{~mm}-\mathrm{KCl}$, $1 \mathrm{~mm}-\mathrm{MgCl}_{2}, 10 \mathrm{~mm}-\mathrm{N}$-acetylcysteine and $0.5 \mathrm{~mm}$-dithiothreitol. After centrifugation at $100000 \mathrm{~g}$ for $40 \mathrm{~min}$ at $4^{\circ} \mathrm{C}$, the supernatant fraction was used for quantitation of the enzyme activities. Fatty acid synthase (FAS, EC 2.3.1.85), glucose-6phosphate dehydrogenase (G6PDH, EC 1.1.1.49), malic enzyme (EC 1.1.1.40) and acetyl-CoA carboxylase (ACC, EC 6.4.1.2) activities were measured as previously described ${ }^{(21)}$ Enzyme activities were expressed either as nmol NADPH consumed (FAS), nmol NADPH produced (G6PDH and malic enzyme) or nmol $\mathrm{HCO}_{3}^{-}$incorporated (ACC), per min, per mg of protein.

Carnitine palmitoyltransferase-Ia (CPT-Ia) and ACO activities were assessed in the mitochondrial/peroxisomal fraction. Liver samples $(500 \mathrm{mg})$ were homogenised in 3 volumes (wt/vol) of buffer ( $\mathrm{pH} 7 \cdot 4$ ) containing $0.25 \mathrm{~mol} / 1$ sucrose, $1 \mathrm{~mm}$-EDTA and $10 \mathrm{~mm}$-Tris-HCl. Homogenates were centrifuged $\left(700 \mathrm{~g}\right.$ for $10 \mathrm{~min}$ at $4^{\circ} \mathrm{C}$ ) and supernatant fluid was again centrifuged $\left(12000 \mathrm{~g}\right.$ for $15 \mathrm{~min}$ at $4^{\circ} \mathrm{C}$ ). Pellets were resuspended in $70 \mathrm{~mm}$-sucrose, $220 \mathrm{~mm}$-mannitol, $1 \mathrm{~mm}$-EDTA, 2 mM-HEPES buffer (pH 7·4). CPT-I activity was assayed by using Bieber et al. ${ }^{(22)}$ method and ACO activity was assayed following the Lazarow method ${ }^{(23)}$. The pellet protein content was determined according to the Bradford method ${ }^{(24)}$. CPT-I activity was expressed as nmol CoA formed/min per $\mathrm{mg}$ protein and ACO activity as nmol $\mathrm{NADH}$ formed/min per $\mathrm{mg}$ protein.

\section{Oxidative stress parameters}

Lipid peroxidation was determined by measuring the formation of thiobarbituric acid reactive substrates TBARS (TBARS Assay Kit; Cayman Chemical Company, Ann Arbor, MI, USA) in liver homogenates. Concentrations were measured against a standard curve obtained with malonaldehyde. TBARS values were expressed as $\mu \mathrm{m}$ malonaldehyde/mg protein.

GSH and GSSG levels were determined spectrophotometrically by using a commercial kit (Glutathione Detection Kit; Assay Designs, Plymouth Meeting, PA, USA). The amounts of 
Table 1. Final body weight, body weight increase, food intake and adipose tissue weights of obese Zucker rats treated or not treated with resveratrol $(15 \mathrm{mg} / \mathrm{kg}$ body weight per d in RSV 15 and $45 \mathrm{mg} / \mathrm{g}$ body weight per d in RSV45) for 6 weeks

(Mean values with their standard errors, $n$ 10)

\begin{tabular}{|c|c|c|c|c|c|c|c|}
\hline & \multicolumn{2}{|c|}{ Control } & \multicolumn{2}{|c|}{ RSV15 } & \multicolumn{2}{|c|}{ RSV45 } & \multirow[b]{2}{*}{$P \S$} \\
\hline & Mean & SEM & Mean & SEM & Mean & SEM & \\
\hline Final body weight (g) & 468 & 9 & $415^{\star \star \star}$ & 7 & $436 \dagger$ & 13 & $<0.05$ \\
\hline Body weight increase (g) & 251 & 6 & $201^{\star \star *}$ & 7 & 226††‡ & 9 & $<0.05$ \\
\hline $\begin{array}{l}\text { Food intake }(\mathrm{g} / \mathrm{d}) \\
\text { Adipose tissue wejahts (d) }\end{array}$ & $32 \cdot 6$ & 0.5 & $28 \cdot 6$ & 0.6 & $30 \cdot 6$ & 0.8 & NS \\
\hline $\mathrm{EP}+\mathrm{PR}+\mathrm{MS}$ & $17 \cdot 6$ & 0.6 & $14 \cdot 9^{* \star}$ & 0.4 & $15 \cdot 4 \dagger$ & 0.5 & $<0.01$ \\
\hline Subcutaneous & $45 \cdot 2$ & $3 \cdot 8$ & $41 \cdot 8$ & $2 \cdot 0$ & $40 \cdot 1$ & $2 \cdot 7$ & NS \\
\hline
\end{tabular}

EP, epididymal; PR, perirenal; MS, mesenteric.

Values were significantly different (Newman-Keuls test): control $v$. RSV15 $\left.{ }^{\star \star} P<0.01 ;{ }^{\star \star \star} P<0.001\right)$; control $v$. RSV45 († $P<0.05 ;$ † $P<0.01)$; RSV15 v. RSV45 ( $P<0.05)$.

$\S$ Values were significantly different among groups (ANOVA).

GSH and GSSG were calculated from a standard curve and were expressed in $\mu \mathrm{m} / \mathrm{mg}$ protein. The redox index was calculated by dividing the concentration of GSH by that of GSSG.

For superoxide dismutase (SOD) assessment a commercial kit was also used (Superoxide Dismutase Activity Assay Kit; BioVision Research Products, Mountain View, CA, USA).

\section{Serum parameters}

For the evaluation of serum parameters commercial kits were used: NEFA (Roche Diagnostics GmbH, Mannheim, Germany); TAG; total cholesterol and HDL-cholesterol (BioSystems, Barcelona, Spain), glycerol (BioVision Research Products); ketone bodies (Roche Diagnostics $\mathrm{GmbH}$ ); glucose (BioSystems); insulin (EZRMI 13K; Linco, St Charles, MO, USA); aspartate aminotransferase (AST/GOT); alanine aminotransferase (ALT/GPT); alkaline phosphatase (Spinreact) activities, and adiponectin (catalogue no. EZRADP-62K; Millipore Iberica, Madrid, Spain). Cholesterol, TAG, AST, ALT and alkaline phosphatase levels were determined in fresh samples and other parameters were assessed in frozen samples.

Non-HDL-cholesterol was calculated by the difference between total cholesterol and HDL-cholesterol.

\section{Statistical analysis}

Results are presented as means with their standard errors. Statistical analysis was performed using SPSS 17.0 (SPSS, Inc., Chicago, IL, USA). Data were analysed by ANOVA I test and Newman-Keuls as post hoc test. Statistical significance was set up at the $P<0.05$ level.

\section{Results}

\section{Body weight, food intake and white adipose tissue weights}

Rats treated with resveratrol showed significantly reduced final body weight $(P<0.05)$, without significant changes in food intake. The reduction in body weight was in part accounted for by a reduction in adipose tissue. More specifically, a significant reduction was observed in internal depots (perirenal + epididymal + mesenteric; $P<0 \cdot 01)$. No significant differences were found between both resveratrol doses (Table 1).

\section{Liver weight and fat content}

Resveratrol significantly reduced liver weight $(P<0.001$; Fig. 1(A)). Hepatic index, obtained by dividing liver weight by body weight $\times 100$, was also lower in rats supplemented with resveratrol $(P<0 \cdot 05$; Fig. $1(\mathrm{~B}))$. TAG content was significantly reduced by resveratrol when expressed either as $\mathrm{mg} / \mathrm{g}$
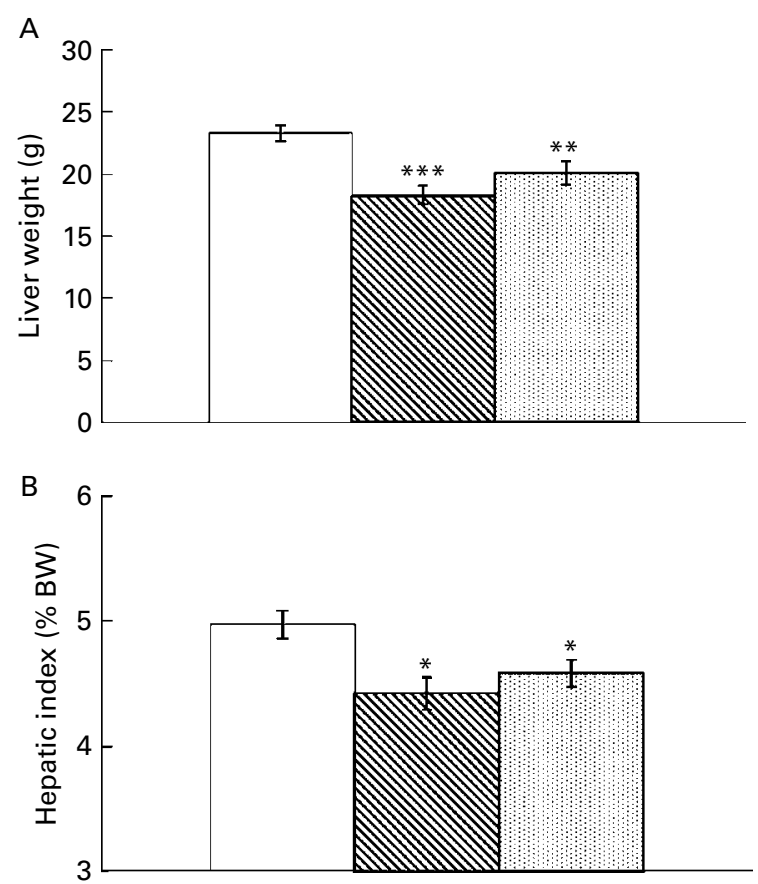

Fig. 1. (A) Liver weight and (B) hepatic index (expressed as percentage of body weight $(\mathrm{BW})$ ) in obese Zucker rats either treated or not treated with resveratrol $(15 \mathrm{mg} / \mathrm{kg}$ body weight per d in RSV15 $(\mathbb{\$})$ and $45 \mathrm{mg} / \mathrm{kg}$ body weight per d in RSV45 ( $\square$ )) for 6 weeks. Values are means, with their standard errors represented by vertical bars, $n 10$. Mean values were significantly different: ${ }^{*} P<0.05,{ }^{\star \star} P<0.01,{ }^{\star \star \star} P<0.001$. $\square$, Control. 

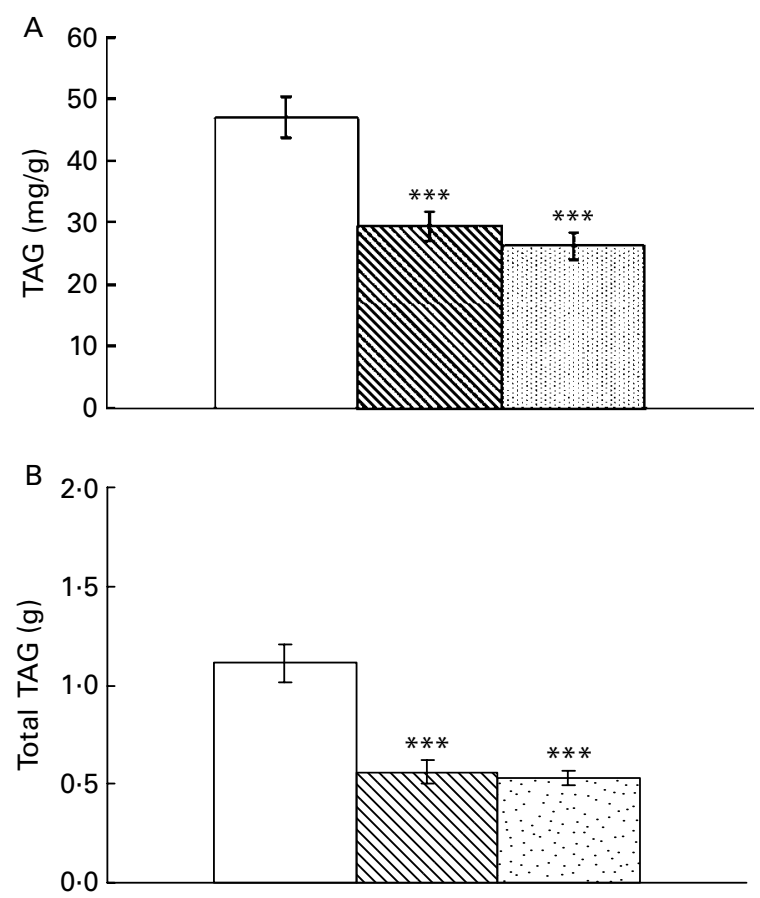

Fig. 2. (A) Hepatic TAG content expressed as $\mathrm{mg} / \mathrm{g}$ tissue or (B) total amount in obese Zucker rats either treated or not treated with resveratrol ( $15 \mathrm{mg} / \mathrm{kg}$ body weight per d in RSV15 (\$) and $45 \mathrm{mg} / \mathrm{kg}$ body weight per $\mathrm{d}$ in RSV45 ( $\square$ )) for 6 weeks. Values are means, with their standard errors represented by vertical bars, $n 10$. Mean values were significantly different: ${ }^{* * \star} P<0.001$. $\square$, Control.

tissue $(P<0.001)$ or as total amount $(\mathrm{g})(P<0 \cdot 001$; Fig. 2(A) and (B)) but no differences were found between both doses. No significant changes were induced by resveratrol in cholesterol content $(6.74$ (SEM 0.44$) \mathrm{mg} / \mathrm{g}$ in the control group, 6.73 (SEm 0.46$) \mathrm{mg} / \mathrm{g}$ in the RSV 15 group and 5.82 (sEm $0 \cdot 27) \mathrm{mg} / \mathrm{g}$ in the RSV45 group).

Mean fat infiltration in the non-treated rats was 3. Fat depot in this group was classified as macrovesicular. Mean fat infiltration in the RSV15 group was 1, and fat depot was mixed. Finally, fat infiltration in the RSV45 group was 2 (Fig. 3).
Inter-observer agreement was 0.84 and intra-observer agreement was $0 \cdot 79$.

\section{Enzyme activities}

No significant differences in the activity of lipogenic enzymes were found among the three experimental groups (Fig. 4). On the contrary, resveratrol significantly increased CPT-Ia activity $(P<0.05)$ and ACO activity $(P<0.05)$ in both RSV groups (Fig. 5).

\section{Serum parameters}

Resveratrol significantly reduced concentration of NEFA $(P<0 \cdot 001)$ and total cholesterol $(P<0 \cdot 01)$. In the RSV15 group levels of both HDL-cholesterol and non-HDL-cholesterol were significantly decreased $(P<0 \cdot 01)$, but in the RSV45 group only the HDL fraction was reduced $(P<0 \cdot 01)$. Although levels of serum TAG were not significantly modified, a trend $(P=0 \cdot 07)$ towards reduced values was observed in the RSV15 group. Concentrations of glucose, glycerol and ketone bodies remained unchanged. Regarding parameters related to liver damage, values of AST/GOT, ALT/GPT and alkaline phosphatase were significantly reduced by the lowest dose $(P<0 \cdot 05)$. A trend towards lower values was observed with the high dose $(P=0.07)$ in transaminases. Although statistical significance was not reached in insulin concentrations, a clear tendency $(P=0.057)$ towards increased values was found in the resveratrol-treated groups. No changes were observed in adiponectin (Table 2).

\section{Oxidative stress}

Resveratrol significantly decreased hepatic TBARS formation, indicating an antioxidant effect and protection from the oxidative stress induced by obesity and steatosis in Zucker rats (Table 3). Also, the high dose was able to diminish the amount of GSSG as well as to increase the GSH:GSSG ratio,
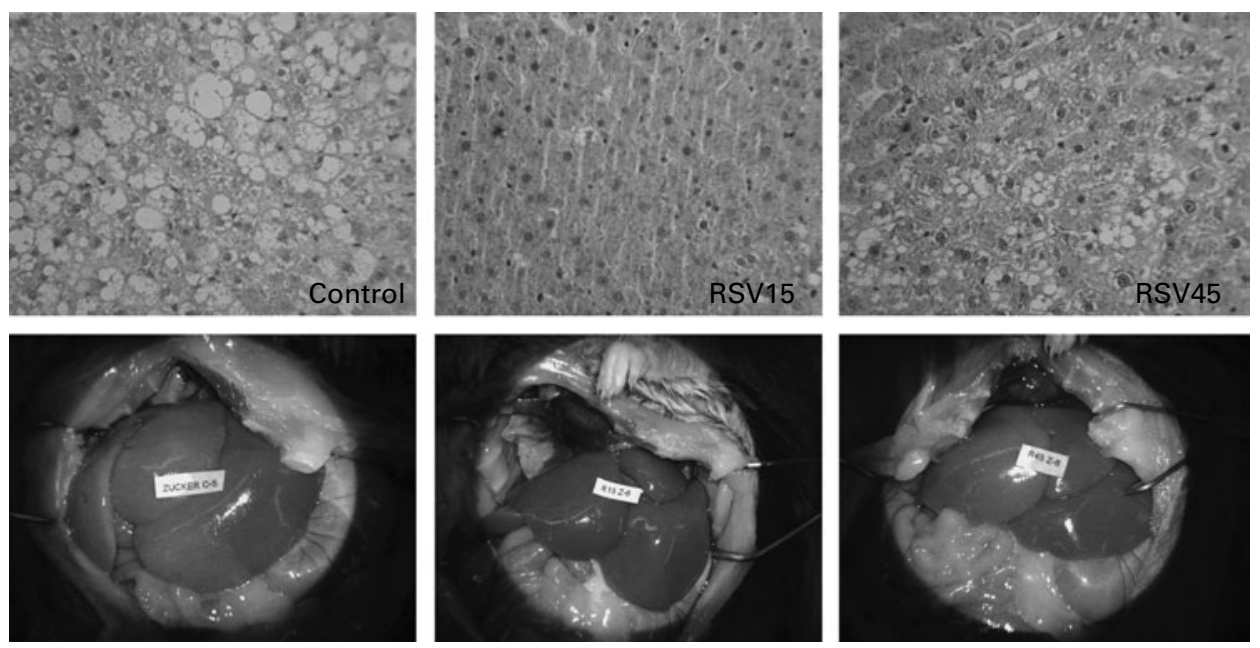

Fig. 3. Histological study in liver from obese Zucker rats either treated or not treated with resveratrol $(15 \mathrm{mg} / \mathrm{kg}$ body weight per $\mathrm{d}$ in RSV15 and $45 \mathrm{mg} / \mathrm{kg}$ body weight per d in RSV45) for 6 weeks. Haematoxylin and eosin staining of liver tissue $\times 40$. 


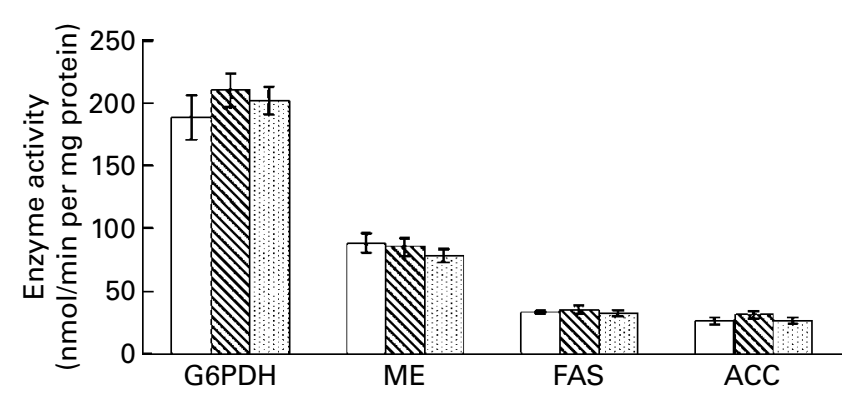

Fig. 4. Glucose-6P-dehydrogenase (G6PDH), malic enzyme (ME), fatty acid synthase (FAS) and acetyl-CoA carboxylase (ACC) activities in liver from obese Zucker rats either treated or not treated with resveratrol $(15 \mathrm{mg} / \mathrm{kg}$ body weight per d in RSV15 ( $\$$ ) and $45 \mathrm{mg} / \mathrm{kg}$ body weight per d in RSV45 (圆)) for 6 weeks. Values are means, with their standard errors represented by vertical bars, $n$ 10. $\square$, Control.

a sensitive and reliable measure of the overall level of oxidative stress (Table 3). These results suggest that the glutathione redox state has become less pro-oxidising due to supplementation with resveratrol. However, the reactive oxygen species scavenging enzyme superoxide dismutase seems not to be involved in the resveratrol-induced reduction of oxidative stress.

\section{Discussion}

Resveratrol has been reported to have significant effects on lipid metabolism. In this context, several studies have shown reduced fat accumulation either in isolated adipocytes or in adipose tissue ${ }^{(11,13)}$ and liver ${ }^{(14,16,25)}$, increased fatty acid oxidation in skeletal muscle $(10,11)$ and reduced serum lipids ${ }^{(16)}$ induced by this polyphenol. All these effects support the hypothesis that resveratrol could be a useful molecule for the prevention of obesity, steatosis and dyslipidaemia.

In the present study, the effects of resveratrol on serum lipids, liver lipids and body fat accumulation have been assessed in $\mathrm{fa} / \mathrm{fa}$ Zucker rats, an animal model that shows obesity, liver steatosis and dyslipidaemia. Nevertheless, as described in the beginning, the main purpose of the study was to analyse the effects of this polyphenol on liver TAG metabolism.

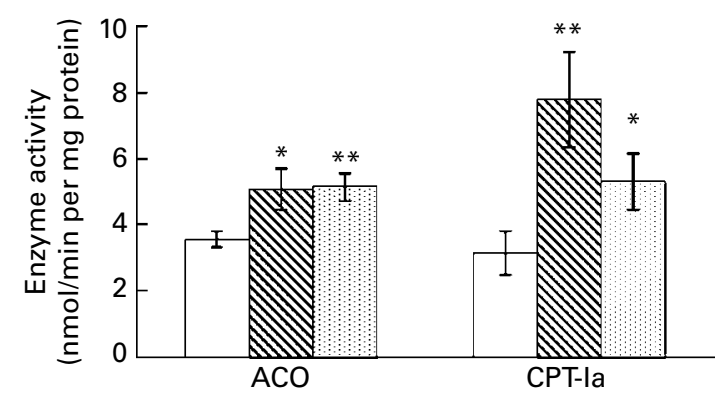

Fig. 5. Acyl-coenzyme A oxidase (ACO) and carnitine palmitoyltransferase-la (CPT-la) activities in liver from obese Zucker rats either treated or not treated with resveratrol $(15 \mathrm{mg} / \mathrm{kg}$ body weight per d in RSV15 (\$) and $45 \mathrm{mg} / \mathrm{kg}$

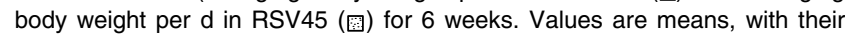
standard errors represented by vertical bars, $n 10$. Mean values were significantly different: ${ }^{\star} P<0.05,{ }^{\star \star} P<0.01$. $\square$, Control.
Liver in obese Zucker rat shows an increased lipogenesis, potentiated by a huge glycolytic flux that leads to the formation of reduction potential in the form of NADPH, and a strongly reduced fatty acid oxidation. Plasma concentrations of glycerol and NEFA are high, which represents an increased flux of these metabolic substrates to the liver for TAG synthesis. Taken together, these alterations lead to steatosis ${ }^{(4)}$.

In the present study, resveratrol treatment resulted in a significant reduction in liver weight. This was not just a consequence of the generalised reduction in body weight, but a direct effect on the organ because significant differences were also observed in the hepatic index (percentage of liver weight with regard to final body weight).

The decrease in liver weight was partly due to a reduction in TAG, as revealed by the assessment of the histological analysis. This is a semi-quantitative system referred to as 'grading' of steatosis ${ }^{(26)}$. Quantifying fat biochemically is a more objective and accurate method of determining the fat content in liver tissue. Thus, we also analysed the amount of liver TAG by using a spectrophotometric method. The results confirmed the de-lipidating effect of resveratrol. This effect is in good accordance with other published studies performed either in mice $^{(10,12,13)}$ or in rats ${ }^{(14,16,25)}$.

Taking into account that lipogenesis and fatty acid oxidation are two key metabolic pathways in the control of hepatic TAG metabolism and hence in fat accumulation in the liver, and considering that both of them are altered in obese Zucker rats, the effects of resveratrol on the activity of several enzymes involved in these pathways were investigated to assess the mechanisms by which this polyphenol decreased liver TAG content. To the best of our knowledge, this is the first paper that analyses the effects of resveratrol on the balance between fatty acid oxidation and activities of lipogenesis enzyme in the liver, to explain its beneficial effects on hepatic steatosis.

As far as lipogenic enzymes are concerned, no changes were observed in the activities of G6PDH and malic enzyme, the enzymes involved in the production of NADPH, or in ACC and FAS. No data concerning the effects of resveratrol on the activity of G6PDH, malic enzyme and FAS in liver have been reported in the literature. On the contrary, the effect of resveratrol of FAS mRNA levels has been addressed. Shang et al. ${ }^{(14)}$ reported that levels of FAS mRNA were significantly reduced in rats treated with resveratrol $(100 \mathrm{mg} / \mathrm{kg}$ body weight) for 16 weeks. The results in the present study are not in good accordance with those reported by Shang et $a l .{ }^{(14)}$. The discrepancy between the lack of effect on FAS activity in the present study and the reduction in the gene expression in the reported study can be due to important differences in experimental design. Whereas in the study reported by Shang et $a l^{(14)}$ the dose of resveratrol was $100 \mathrm{mg} / \mathrm{kg}$ body weight and the length of the treatment was 16 weeks, in the present study lower doses $(15$ and $45 \mathrm{mg} / \mathrm{kg}$ body weight) and a shorter experimental period ( 6 weeks) were used. It can be hypothesised that FAS needs high doses of resveratrol and/or longer treatments to be modified. Moreover, whereas in the study reported by Shang et al. ${ }^{(14)}$ resveratrol was administered $4 \mathrm{~h}$ before killing, in the present 
Table 2. Serum parameters of obese Zucker rats treated or not treated with resveratrol $(15 \mathrm{mg} / \mathrm{kg}$ body weight per d in RSV15 and $45 \mathrm{mg} / \mathrm{g}$ body weight per d in RSV45) for 6 weeks

(Mean values with their standard errors, $n 10$ )

\begin{tabular}{|c|c|c|c|c|c|c|c|}
\hline & \multicolumn{2}{|c|}{ Control } & \multicolumn{2}{|c|}{ RSV15 } & \multicolumn{2}{|c|}{ RSV45 } & \multirow[b]{2}{*}{$P \S$} \\
\hline & Mean & SEM & Mean & SEM & Mean & SEM & \\
\hline $\mathrm{NEFA}(\mathrm{mmol} / \mathrm{l})$ & 0.58 & 0.05 & $0.38^{* \star *}$ & 0.05 & $0.28 \dagger \dagger \dagger$ & 0.03 & $<0.001$ \\
\hline Total cholesterol (mg/l) & 2060 & 60 & $1720^{\star *}$ & 500 & $1800+\dagger$ & 60 & $<0.01$ \\
\hline HDL-cholesterol (mg/l) & 1360 & 40 & $1170^{\star \star}$ & 30 & $1080+\dagger \dagger$ & 40 & $<0.01$ \\
\hline Non-HDL-cholesterol (mg/l) & 700 & 30 & $550^{\star *}$ & 30 & $720 \ddagger \ddagger$ & 50 & $<0.01$ \\
\hline TAG (mg/l) & 2800 & 280 & 2120 & 210 & 2500 & 360 & NS \\
\hline Glycerol (mmol/l) & 0.24 & 0.01 & 0.20 & 0.02 & 0.23 & 0.03 & NS \\
\hline Ketone bodies $(\mathrm{mmol} / \mathrm{l})(\mathrm{AcAc}+\beta-\mathrm{HB})$ & 0.70 & 0.14 & 0.60 & 0.08 & 0.82 & $0 \cdot 10$ & NS \\
\hline Glucose $(\mathrm{mg} / \mathrm{l})$ & 1430 & 100 & 1690 & 70 & 1600 & 90 & NS \\
\hline Insulin (pmol/l) & 861 & 119 & $1316^{*}$ & 175 & $1309+$ & 112 & $<0.05$ \\
\hline Adiponectin $(\mu \mathrm{g} / \mathrm{ml})$ & 8.00 & 1.00 & $9 \cdot 17$ & 0.55 & $8 \cdot 72$ & 0.90 & NS \\
\hline AST/GOT (U/I) & 193 & 18 & $138^{* *}$ & 12 & 153 & 10 & $<0.05$ \\
\hline ALT/GPT (U/I) & 133 & 8 & $94^{\star *}$ & 9 & 113 & 6 & $<0.05$ \\
\hline $\operatorname{ALP}(\mathrm{U} / \mathrm{I})$ & 154 & 9 & $124^{\star *}$ & 5 & $142 \ddagger$ & 7 & $<0.05$ \\
\hline
\end{tabular}

study the last resveratrol administration took place the day before killing. Taking into account the short half-life of this polyphenol $^{(27)}$, this is an important difference between both studies. Ahn et al. ${ }^{(13)}$ found the same effects in the liver of a mice who was fed a diet enriched with resveratrol (0.0125\%) for 8 weeks. Once again this experimental period is longer than the one used in the present study. Moreover, mice are the most sensitive species with regard to the effects of some functional ingredients. This has been demonstrated, for instance, in the case of conjugated linoleic acid ${ }^{(28)}$.

It should be also pointed out that the reduction in FAS mRNA levels observed by Shang et al. ${ }^{(14)}$ was probably related to the decrease in serum insulin induced by resveratrol that they observed. In the present study, serum insulin concentrations in resveratrol-treated groups showed a clear tendency towards increased values. The effect of resveratrol on insulin levels is unclear. Thus, while several authors have reported decreased values of serum insulin in resveratrol-treated animals, others authors have found increased values ${ }^{(29)}$. Nevertheless, and despite all the mentioned differences, it should be emphasised that while Shan et al. ${ }^{(14)}$ and Ahn et al. ${ }^{(13)}$ evaluated FAS expression, the present study measured FAS activity. It is well known that although one of the most important mechanisms of FAS regulation takes place at the transcriptional level, other post-transcriptional mechanisms also contributes to enzyme activity regulation ${ }^{(30,31)}$.

With regard to ACC, it has been proposed that resveratrol can phosphorylate this enzyme by AMP-activated protein kinase, thus leading to a decrease in its activity. This effect was observed by Rivera et $a l^{(16)}$ in obese Zucker rats treated with resveratrol for 8 weeks and also by Shang et al. ${ }^{(14)}$ in the afore-mentioned paper. In the present study, instead of measuring the phosphorylation of ACC to estimate the activation/deactivation process, we directly measured the activity of the enzyme by radiometry. Interestingly, this activity was not reduced in the rats treated with resveratrol.

To understand these results it is important to remember that the short-term control of ACC activity is achieved not only by phosphorylation/dephosphorylation, but also by allosteric regulation. Citrate and fatty acid concentrations, which can

Table 3. Oxidative stress parameters in liver of obese Zucker rats treated or not treated with resveratrol $(15 \mathrm{mg} / \mathrm{kg}$ body weight per $\mathrm{d}$ in RSV15 and $45 \mathrm{mg} / \mathrm{g}$ body weight per d in RSV45) for 6 weeks

(Mean values with their standard errors, $n$ 10)

\begin{tabular}{|c|c|c|c|c|c|c|c|}
\hline & \multicolumn{2}{|c|}{ Control } & \multicolumn{2}{|c|}{ RSV15 } & \multicolumn{2}{|c|}{ RSV45 } & \multirow[b]{2}{*}{$P \S$} \\
\hline & Mean & SEM & Mean & SEM & Mean & SEM & \\
\hline TBARS ( $\mu \mathrm{M}$ MAD/mg protein) & $90 \cdot 2$ & 8.2 & $64 \cdot 2^{* *}$ & $5 \cdot 2$ & $67.5 \dagger$ & 3.5 & $<0.05$ \\
\hline Total glutathione ( $\mu \mathrm{M} / \mathrm{mg}$ protein) & 21.0 & $2 \cdot 8$ & 18.6 & 1.7 & $16 \cdot 5$ & 1.8 & NS \\
\hline GSSG ( $\mu \mathrm{M} / \mathrm{mg}$ protein) & 1.0 & 0.1 & 0.7 & 0.1 & $0.3+† \dagger \ddagger$ & 0.1 & $<0.05$ \\
\hline GSH ( $\mu \mathrm{M} / \mathrm{mg}$ protein) & $20 \cdot 0$ & $2 \cdot 7$ & 17.9 & 1.7 & $16 \cdot 2$ & 1.7 & NS \\
\hline GSH:GSSG ratio & $20 \cdot 4$ & $2 \cdot 0$ & 33.4 & 7.6 & 58.7†††‡ & $6 \cdot 0$ & $<0.05$ \\
\hline SOD ( $\mu \mathrm{M} / \mathrm{mg}$ protein) & 1643 & 115 & 1571 & 301 & 1678 & 64 & NS \\
\hline
\end{tabular}

MAD, malonaldehyde; SOD, superoxide dismutase.

Values were significantly different between groups (Newman-Keuls test): control $v$. RSV15 ${ }^{\star *} P<0.01$ ); control $v$. RSV45 († $P<0.05$; ††† $P<0.001$ ); RSV15 v. RSV45 ( $¥ P<0.05$ )

$\S$ Values were significantly different among groups (ANOVA). 
perturb the equilibrium of ACC polymerisation process, are the main molecules responsible for allosteric regulation ${ }^{(32)}$. Citrate activates ACC by causing its polymerisation. Contrastingly, long-chain fatty acyl CoA esters are potent inhibitors of ACC because they promote the dissociation of the polymer into protomers ${ }^{(33)}$

In the present study, the concentrations of these molecules were not measured but, as stated further on in this discussion, fatty acid availability seems to decrease in the liver of rats treated with resveratrol. Thus, a compensation between ACC inhibition, due to an increased phosphorylation, and the enzyme activation, due to the reduction of the physiological inhibitor (fatty acyl-CoA), probably led to the lack of change when the activity of this enzyme was directly measured.

Altogether these results show that, under the present experimental conditions, a reduction in de novo lipogenesis did not contribute to the reduction in liver TAG induced by resveratrol.

The effect of resveratrol on the activities of CPT-Ia, a key enzyme in mitochondrial fatty acid oxidation, and ACO, a key enzyme in peroxisome fatty acid oxidation, was also assessed in the present study. Resveratrol treatment resulted in increased activity of both enzymes. It has been proposed that resveratrol, by activation of sirtuins ${ }^{(34,35)}$, deacetylates and thus activates the peroxisome proliferator-activated receptor- $\gamma$ coactivator- $1^{(18)}$, leading to increased mitochondrial activity and function ${ }^{(10,18,36)}$. The increased CPT-Ia activity observed in the present study supports this hypothesis. The lack of changes in serum ketone bodies is noteworthy. Perhaps the increase in enzyme activity was not high enough to enhance this parameter. A similar situation has been reported by other authors. Stefanovic-Racic et al. ${ }^{(37)}$ observed that $60 \%$ over-expression of CPT-Ia in liver resulted in a significant increase in the rate of fatty acid oxidation (45\%) and a significant decrease in TAG content (70\%), without changes in plasma ketone bodies.

Adiponectin appears to have a pivotal role in improving fatty acid oxidation and decreasing fatty acid synthesis ${ }^{(38)}$. The liver has adiponectin receptors, and their stimulation leads to increased fatty acid $\beta$-oxidation and thereby decreased hepatic TAG content. In the present study, despite the significant reduction induced by resveratrol in fat depot weights, no changes were observed in serum adiponectin concentrations among the three experimental groups, meaning that the effect of resveratrol on liver fatty acid oxidation was not mediated by this adipokine.

As stated in the beginning, plasma NEFA levels are high in obese Zucker rats because of increased adipose tissue mass and peripheral insulin resistance. These lipids can enter the hepatocyte and there they play an important role in stimulating hepatic TAG production ${ }^{(39)}$. Inside the hepatocytes NEFA can be either oxidised or esterified. In obese Zucker rats, enhanced esterification and reduced oxidation leads to ectopic deposition of TAG in the liver. In the present study resveratrol significantly reduced serum NEFA. This effect was probably related to the observed reduction in adipose tissue mass $(-15 \cdot 3 \%$ and $-12.5 \%$ in internal depots for the RSV15 and RSV45 groups, respectively, and -7.5 and $-11.3 \%$ in the subcutaneous depot for the RSV15 and RSV45 groups, respectively). Nevertheless, the involvement of a potential reduction in the ex vivo lipoprotein lipase-dependent VLDLTAG hydrolysis cannot be discarded ${ }^{(40)}$ because in the present experimental design blood collected was not treated with antilipolytic agents to block this process. Although NEFA flux into the liver was not directly assessed in the present study, it could be hypothesised that it may be reduced.

Collectively, these results demonstrate that resveratrol reduces the availability of NEFA, an important substrate for TAG synthesis in the liver, by increasing their oxidation and probably by reducing their flux from plasma, thus resulting in a decrease in fat accumulation.

Both mitochondrial and peroxisomal fatty acid oxidation are capable of producing hepatotoxic free oxygen radicals that contribute to the development of oxidative stress ${ }^{(41)}$, an imbalance between oxidants and antioxidants systems in favour of the former. Moreover, it has been demonstrated that oxidative stress can, at least to some extent, be responsible for further progression from steatosis to steatohepatitis and fibrosis ${ }^{(42,43)}$. In the present study, fatty acid oxidation was likely to increase as a consequence of enhanced CPT-Ia and ACO activities. In this context, the levels of oxidative stress are a matter of concern. With regard to this issue, it should be pointed out that resveratrol has been reported to show antioxidant properties, but it is important not to forget that resveratrol, as well as other antioxidants, can become pro-oxidant at high doses ${ }^{(17,44,45)}$.

To shed light onto this situation, we analysed several markers of hepatic oxidative stress. The biological antioxidant defence system is an integrated array of enzymes and antioxidants $^{(46)}$. TBARS levels are one of the most extensively used markers of lipid peroxidation and oxidative damage. Moreover, the glutathione antioxidant system is considered very efficient at decreasing the presence of free radicals. GSH is required to maintain the normal reduced state and to counteract the deleterious effects of oxidative stress. It is commonly recognised that reduction in GSH:GSSG ratio denotes the presence of oxidative stress. Superoxide dismutase is the enzyme that catalyses the destruction of $\mathrm{O}_{2}^{-}$by dismutation and $\mathrm{H}_{2} \mathrm{O}_{2}$ formation.

The present results show that resveratrol administration was able to reduce the oxidative damage in liver, measured by TBARS and the amount of GSSG, although not by superoxide dismutase. With this mechanism, resveratrol can limit the progression of liver steatosis. As previously stated in this section, resveratrol, as well as other antioxidants, can become pro-oxidant at high doses. The present results show that in the range of $15-45 \mathrm{mg} / \mathrm{kg}$ body weight, this polyphenol maintains its antioxidant properties.

The results reported here show that resveratrol has a beneficial effect on liver in obese Zucker rats because it attenuates steatosis and decreases oxidative stress. The suspicion of NAFLD is usually prompted by abnormal serum liver biochemical findings. Usually the levels of ALT, AST or both are increased mildly to moderately. In the present study, control animals showed high levels of both transaminases, in 
comparison with published values in normal rats not showing steatosis $^{(26,47)}$, in good accordance with the presence of fatty liver in these animals. Resveratrol induced a reduction in AST, ALT and alkaline phosphatase levels that reached statistical significance in RSV15 group and remained as a tendency in RSV45 group. Both cholesterol and TAG contribute to liver damage, but in the present study changes in transaminases were only related to the decrease in TAG content because cholesterol content remained unchanged.

While liver cholesterol was not modified, serum total cholesterol was significantly reduced in resveratrol-treated rats. Both HDL-cholesterol and non-HDL-cholesterol were decreased in the RSV15 group and HDL-cholesterol in the RSV45 group. Several mechanisms could be involved in these effects: (1) reduced liver cholesterogenesis, a metabolic process that is over-stimulated in obese Zucker rats ${ }^{(4)}$, according to the reduction in the expression of hydroxymethylglutaryl CoA reductase reported by Cho et al. ${ }^{(48)}$; (2) decreased endogenous cholesterol re-absorption ${ }^{(49)}$; (3) increased excretion of bile acids into faeces ${ }^{(50)}$ and (4) decreased HDL stability, as reported by Noll et al. ${ }^{(51)}$.

In general terms, a dose-response pattern was not found, and only punctual differences were found between both resveratrol doses in several parameters. This suggests that a 'plateau' is apparently reached when the beneficial effects of this polyphenol on fatty liver are considered, and thus that the high dose provides no additional benefit over the lowest dose.

In conclusion, this study demonstrates that resveratrol can protect the liver from NAFLD by reducing fatty acid availability. Moreover, it protects this organ from oxidative stress.

\section{Acknowledgements}

This study was supported by grants from the Ministerio de Ciencia e Innovación (AGL2008-1005-ALI), Instituto de Salud Carlos III (RETIC PREDIMED and CIBERehd) and Government of País Vasco (IT-386-10; CTP09/R5). S. G. Z. is a recipient of a doctoral fellowship from the University of País Vasco. S. G. Z., A. F. Q. and M. T. M. measured enzyme activities and plasma metabolites; L. A. and F. M. carried out oxidative stress measurements; E. H. and L. B. treated the rats and analysed histological preparations; M. T. M., J. A. M. and M. P. P. designed the research; M. P. P. wrote the paper and had the responsibility for the final content. All authors read and approved the final manuscript. No conflicts of interest are reported by any of the authors.

\section{References}

1. Bellentani S \& Tiribelli C (2001) The spectrum of liver disease in the general population: lessons from Dyonisos study. J Hepatol 35, 531-537.

2. Fabrini E, Sullivan S \& Klein S (2010) Obesity and nonalcoholic fatty liver disease: biochemical, metabolic and clinical implications. Hepatology 51, 679-689.

3. Marchesini G, Bugianesi E, Forlani G, et al. (2003) Nonalcoholic fatty liver, steatohepatitis and the metabolic syndrome. Hepatology 37, 917-923.
4. Argiles JM (1989) The obese Zucker rat: a choice for fat metabolism. Pro Lipid Res 28, 33-66.

5. Langcake P \& Pryce RJ (1974) The production of resveratrol by Vitis vinifera and other members of the Vitaceae as a response to infection or injury. Physiol Plant Phatol 9 , 77-86.

6. Signorelli P \& Ghidoni R (2005) Resveratrol as an anticancer nutrient: molecular basis, open questions and promises. J Nutr Biochem 16, 449-466.

7. Frémont L (2000) Biological effects of resveratrol. Life Sci 66, 663-673.

8. Goswami SK \& Das DK (2009) Resveratrol and chemoprevention. Cancer Lett 284, 1-6.

9. Cucciolla V, Borriello A, Oliva A, et al. (2007) Resveratrol: from basic science to the clinic. Cell Cycle 6, 2495-2510.

10. Baur JA, Pearson KJ, Price NL, et al. (2006) Resveratrol improves health and survival of mice on a high-calorie diet. Nature 444, 337-342.

11. Lagouge M, Argmann C, Gerhart-Hines Z, et al. (2006) Resveratrol improves mitochondrial function and protects against metabolic disease by activating SIRT1 and PGC- $1 \alpha$. Cell 127, 1109-1122.

12. Zang M, Xu S, Maitland-Toolan A, et al. (2006) Polyphenols stimulate AMP-activated protein kinase, lower lipids and inhibit accelerated atherosclerosis in diabetic LDL receptordeficient mice. Diabetes 55, 2180-2191.

13. Ahn J, Cho I, Kim S, et al. (2008) Dietary resveratrol alters lipid metabolism-related gene expression of mice on an atherogenic diet. J Hepatol 49, 1019-1028.

14. Shang J, Chen LL, Xiao F, et al. (2008) Resveratrol improves non-alcoholic fatty acid disease by activating AMP-activated protein kinase. Acta Pharmacol Sin 29, 698-706.

15. Macarulla MT, Alberdi G, Gómez S, et al. (2009) Effects of different doses of resveratrol on body fat and serum parameters in rats fed a hypercaloric diet. $J$ Physiol Biochem 65, 369-376.

16. Rivera L, Morón R, Zarzuelo A, et al. (2009) Long-term resveratrol administration reduces metabolic disturbances and lowers blood pressure in obese Zucker rats. Biochem Pharmacol 77, 1053-1063.

17. Van der Spuy W \& Pretorius E (2009) Is the use of resveratrol in the treatment and prevention of obesity premature? Br J Nutr 22, 111-117.

18. Szkudelska K \& Szkudelski T (2010) Resveratrol, obesity and diabetes. Eur J Pharmacol 635, 1-8.

19. Folch J, Lees M \& Sloane Stanley GH (1957) A simple method for the isolation and purification of total lipides from animal tissues. J Biol Chem 226, 497-509.

20. Brunt EM, Janney CG, Di Bisceglie AM, et al. (1999) Non-alcoholic steatosis: a proposal for grading and staging the histological lesions. Am J Gastroenterol 94, 2467-2474.

21. Zabala A, Churruca I, Macarulla MT, et al. (2004) The trans-10, cis-12 isomer of conjugated linoleic acid reduces hepatic triacylglycerol content without affecting lipogenic enzymes in hamsters. Br J Nutr 92, 383-389.

22. Bieber L, Abraham T \& Helmrath T (1972) A rapid spectrophotometric assay for carnitine palmitoyltransferase. Anal Biochem 50, 509-518.

23. Lazarow P (1981) Assay of peroxisomal beta-oxidation of fatty acids. Methods Enzymol 72, 315-319.

24. Bradford M (1976) A rapid and sensitive method for the quantitation of microgram quantities of protein utilizing the principle of protein-dye binding. Anal Biochem 72, 248-254. 
25. Bujanda L, Hijona E, Larzabal M, et al. (2008) Resveratrol inhibits non-alcoholic fatty liver disease in rats. BMC Gastroenterol 8, 40-48.

26. Kleiner DE, Brunt EM, Natta MV, et al. (2005) Design and validation of a histological scoring system for nonalcoholic fatty liver disease. Hepatology 41, 1313-1321.

27. Bertelli AA, Giovannini L, Stradi R, et al. (1998) Evaluation of kinetic parameters of natural phytoalexin in resveratrol orally administered in wine to rats. Drugs Exp Clin Res 24, 51-55.

28. Zabala A, Churruca I, Macarulla MT, et al. (2004) The trans10 , cis-12 isomer of conjugated linoleic acid reduces heaptic triacylglycerol content without affecting lipogenic enzymes in hamsters. Br J Nutr 92, 282-289.

29. Szkudelska K \& Szkudelski T (2010) Resveratrol, obesity and diabetes. Eur J Pharmacol 635, 1-8.

30. Katsurada A, Iritani N, Fukuda H, et al. (1990) Effects of nutrients and hormones on transcriptional and posttranscriptional regulation of fatty acid synthase in rat liver. Eur J Biochem 190, 427-433.

31. Kim K, Park S \& Kim Y (1992) Regulation of fatty acid synthase at transcriptional and post-transcriptional levels in rat liver. Yonsei Med J 33, 199-208.

32. Munday MR (2002) Regulation of mammalian acetyl CoA carboxylase. Biochem Soc Trans 30, 1059-1064.

33. Tong L (2005) Acetyl-Coenzyme A carboxylase: crucial metabolic enzyme and attractive target for drug discovery. Cell Mol Life Sci 62, 1784-1803.

34. Howitz KT, Bittrman KJ, Cohen HY, et al. (2003) Small molecule activator of sirtuins extends Saccharomyces cerevisiae life span. Nature 425, 191-196.

35. Borra MT, Smith BC \& Denu JM (2005) Mechanism of human SIRT1 activation by resveratrol. J Biol Chem 280, $17187-17195$.

36. Medina-Gómez G, Gray S \& Vidal-Puig A (2007) Adipogenesis and lipotoxicity; role of peroxisome proliferatoractivated receptor $\gamma$ (PPAR $\gamma)$ and PPAR $\gamma$-coactivator-1 (PGC1). Public Health Nutr 10, 1132-1137.

37. Stefanovic-Racic M, Perdomo G, Mantell BS, et al. (2008) A moderate increase in carnitine palmitoyltransferase 1a activity is sufficient to substantially reduce hepatic triglyceride levels. Am J Physiol 294, E969-E977.

38. Xu A, Wang $\mathrm{Y}$, Keshaw $\mathrm{H}$, et al. (2003) The fat-derived hormone adiponectin alleviates alcoholic and nonalcoholic fatty liver diseases in mice. J Clin Invest 112, 91-100.

39. Raz I, Eldor R, Cernea S, et al. (2005) Diabetes: insulin resistance and derangements in lipid metabolism. Cure through intervention in fat transport and storage. Diabetes Metabol Res Rev 21, 3-14.

40. Pruneta V, Autran D, Ponsin G, et al. (2001) Ex vivo measurement of lipoprotein lipase-dependent very low density lipoprotein (VLDL)-triglyceride hydrolysis in human VLDL: an alternative to the postheparin assay of lipoprotein lipase activity? J Clin Endocrinol Metab 86, 797-803.

41. Duvnjak M, Lerotic I, Barsic N, et al. (2007) Pathogenesis and management issues for non-alcoholic fatty liver disease. World J Gastroenterol 13, 4539-4550.

42. Spolarics Z \& Meyenhofer M (2000) Augmented resistance to oxidative stress in fatty rat livers induced by a shortterm sucrose-rich diet. Biochim Biophys Acta 1487, 190-200.

43. Albano E, Mottaran E, Vidali M, et al. (2005) Immune response towards lipid peroxidation products as a predictor of progression of non-alcoholic fatty liver disease to advanced fibrosis. Gut 54, 987-993.

44. Faine LA, Rodrigues HG, Galhardi CM, et al. (2006) Effects of olive oil and its minor constituents on serum lipids, oxidative stress and energy metabolism in cardiac muscle. Can $J$ Physiol Pharmacol 84, 239-245.

45. Valdecantos MP, Pérez-Matute P \& Martínez JA (2009) Obesity and oxidative stress: role of antioxidant supplementation. Rev Invest Clin 61, 127-139.

46. Yu BP (1994) Cellular defenses against damage reactive oxygen species. Physiol Rev 74, 139-162.

47. Thong-Ngam D, Samuhasaneeto S, Kulaputana O, et al. (2007) $N$-acetylcysteine attenuates oxidative stress and liver pathology in rats with non-alcoholic steatohepatitis. $J$ Gastroenterol 14, 5127-5132.

48. Cho IJ, Ahn JY, Kim S, et al. (2008) Resveratrol attenuates the expression of HMG-CoA reductase mRNA in hamsters. Biochem Biophys Res Comm 367, 190-194.

49. Sbarra V, Ristorcelli E, Le Pétit-Thévenin J, et al. (2005) In vitro polyphenol effects on activity, expression and secretion of pancreatic bile salt-dependent lipase. Biochim Biophys Acta 1736, 67-76.

50. Miura D, Miura Y \& Yagasaki K (2003) Hypolipidemic action of dietary resveratrol, a phytoalexin in grapes and red wine, in hepatoma-bearing rats. Life Sci 73, 1393-1400.

51. Noll C, Hamalet J, Ducros V, et al. (2009) Resveratrol supplementation worsen the dysregulation of genes involved in hepatic lipid homeostasis observed in hyperhomocysteinemic mice. Food Chem Toxicol 47, 230-236. 